\title{
The Effects of Ultrasound Frequency on Nanodroplet-Mediated Histotripsy
}

Eli Vlaisavljevich ${ }^{1}$, Omer Aydin $^{1}$, Yasemin Yuksel Durmaz ${ }^{1,2}$, Kuang-Wei Lin ${ }^{1}$, Brian Fowlkes ${ }^{1,3}$, Mohamed ElSayed ${ }^{1,4^{*}}$, Zhen $\mathrm{Xu}^{1,5^{*}}$

1 - Department of Biomedical Engineering, University of Michigan, Ann Arbor, MI

2 - Department of Biomedical Engineering, Istanbul Medipol University, Istanbul, Turkey

3 - Department of Radiology, University of Michigan, Ann Arbor, MI

4 - Macromolecular Science and Engineering Program, University of Michigan, Ann Arbor, MI

5 - Department of Pediatrics and Communicable Diseases, Division of Pediatric Cardiology, University of Michigan, Ann Arbor, MI

* Corresponding Author:

Mohamed E.H. ElSayed, Ph.D.

University of Michigan

Department of Biomedical Engineering

1101 Beal Avenue

Lurie Biomedical Engineering Building

Room 2150

Ann Arbor, MI 48109, USA

Phone: + 1 (734) 615-9404

Fax: + 1 (734) 647-4834

E-mail: melsayed@umich.edu

Web: www.bme.umich.edu/centlab.php
* Corresponding Author:

Zhen Xu, Ph.D.

University of Michigan

Department of Biomedical Engineering

2107 Carl A. Gerstacker Building

2200 Bonisteel Boulevard

Ann Arbor, MI 48109, USA

Phone: + 1 (734) 647-4961

Fax: + 1 (734) 939-1905

E-mail: zhenx@umich.edu

Web:www.bme.umich.edu/labs/xulab/

(C) 2015. This manuscript version is made available under the Elsevier user license

http://www.elsevier.com/open-access/userlicense/1.0/ 


\begin{abstract}
Nanodroplet-mediated histotripsy $(\mathrm{NMH})$ is a targeted ultrasound ablation technique combining histotripsy with nanodroplets that can be selectively delivered to tumor cells for targeted tumor ablation. In a previous study, it was demonstrated that, using extremely short, high-pressure pulses, histotripsy cavitation bubbles were generated in regions containing nanodroplets at significantly lower pressure $(\sim 10.8 \mathrm{MPa})$ than without nanodroplets $(\sim 28 \mathrm{MPa})$ at $500 \mathrm{kHz}$. Furthermore, it was hypothesized that lower frequency would improve the effectiveness of NMH by increasing the size of the focal region, increasing bubble expansion, and decreasing the cavitation threshold. In this study, we investigate the effects of ultrasound frequency $(345 \mathrm{kHz}, 500 \mathrm{kHz}, 1.5 \mathrm{MHz}$, and $3 \mathrm{MHz}$ ) on $\mathrm{NMH}$. First, the $\mathrm{NMH}$ cavitation threshold was measured in tissue phantoms with and without nanodroplets, with results showing that the NMH threshold was significantly below the histotripsy intrinsic threshold at all frequencies. Results further showed that the NMH threshold decreased at lower frequency, ranging from 7.4 $\mathrm{MPa}$ at $345 \mathrm{kHz}$ to $13.2 \mathrm{MPa}$ at $3 \mathrm{MHz}$. In the second part of this study, the effects of frequency on $\mathrm{NMH}$ bubble expansion were investigated, with results demonstrating larger expansion at lower frequency even at a lower pressure. In the final part of this study, the ability of PFP-encapsulated nanodroplets to act as sustainable cavitation nuclei over multiple pulses was investigated, with results showing that the nanodroplets are destroyed by the cavitation process and only function as cavitation nuclei for the first few pulses, with this effect being most pronounced at higher frequencies. Overall, the results of this study support our hypothesis that using a lower frequency will improve the effectiveness of NMH by increasing the size of the focal region, increasing bubble expansion, and decreasing the cavitation threshold.
\end{abstract}

Keywords: Nanodroplet-mediated histotripsy, frequency, cavitation 


\section{Introduction}

Histotripsy is a noninvasive, image-guided tissue ablation method that controllably fractionates soft tissue through cavitation generated by high pressure, short duration ultrasound pulses (Xu et al. 2005b; Parsons et al. 2006a; Roberts et al. 2006). Histotripsy depends on the initiation and maintenance of a dense cavitation bubble cloud to produce mechanical tissue fractionation (Xu et al. 2005a; Parsons et al. 2007). Previous work has shown that, using a 1-2 cycle pulse with a single dominant negative pressure phase, histotripsy bubbles can be reproducibly generated in tissue when the peak negative pressure is raised above the histotripsy intrinsic threshold of 25-30 MPa (Maxwell et al. 2013; Vlaisavljevich et al. 2015a). In order to effectively fractionate tissue into acellular debris, histotripsy requires bubbles to rapidly expand into large cavitation bubbles, often greater than $\sim 50 \mu \mathrm{m}$ in diameter (Parsons et al. 2006a; Xu et al. 2007; Vlaisavljevich et al. 2013a; Vlaisavljevich et al. 2015b). Using a pressure high enough to initiate a bubble cloud, histotripsy has been shown capable of completely fractionating soft tissue into a liquid tissue homogenate with no cellular structures remaining ( $\mathrm{Xu}$ et al. 2005b; Roberts et al. 2006; Hall et al. 2007). Histotripsy is currently being studied for many clinical applications in which non-invasive tissue removal is desired including benign prostatic hyperplasia (Hempel et al. 2011), deep vein thrombosis (Maxwell et al. 2011), congenital heart disease (Xu et al. 2010; Owens et al. 2011), and cancer ablation (Styn et al. 2010; Vlaisavljevich et al. 2013b).

Although histotripsy has shown promise for many clinical applications including tumor ablation, this approach is limited to applications in which the target tissue can be identified and imaged prior to treatment, which is often not feasible in cancer patients with many small tumor nodules and micro-metastases. As a result, our team has developed a new targeted ablation 
approach combining perfluoropentane (PFP) encapsulated nanodroplets with histotripsy (Vlaisavljevich et al. 2013a; Yuksel Durmaz et al. 2014). This nanodroplet-mediated histotripsy $(\mathrm{NMH})$ approach takes advantage of the significantly reduced cavitation threshold of the nanodroplets, allowing for cavitation to be selectively generated only in regions containing the nanodroplets (Vlaisavljevich et al. 2013a). Preparing nanodroplets in the size range of $\sim 100-400$ $\mathrm{nm}$ allows them to diffuse across the leaky tumor vasculature and preferentially accumulate in the tumor, which allows NMH to potentially achieve selective ablation of tumors (Vlaisavljevich et al. 2013a; Yuksel Durmaz et al. 2014). In a previous study (Vlaisavljevich et al. 2013a), the initial feasibility of this approach was demonstrated, with results supporting our hypothesis that nanodroplets significantly decrease the histotripsy threshold to form a cavitation bubble cloud while maintaining the effectiveness of histotripsy tissue ablation. NMH was shown capable of creating microbubble expansion and collapse as well as well-defined ablation similar to histotripsy but at significantly lower pressure (Vlaisavljevich et al. 2013a). Furthermore, the potential to use this approach for simultaneous multi-focal ablation was demonstrated (Vlaisavljevich et al. 2013a).

To build upon our initial study, this work aims to investigate the effects of ultrasound frequency on NMH. We hypothesize that lower frequency will offer multiple advantages for NMH therapy. First, as the transducer focal zone scales with the wavelength, a low frequency transducer will yield a large focal zone, allowing histotripsy to be applied simultaneously to cover large and/or multi-nodule tumors seeded with our nanodroplets, thus increasing the treatment efficiency for such tumors. Lower frequency is also more resistant to acoustic aberration and attenuation from bone obstruction and long overlying tissue, resulting in deeper penetration depth. The use of nanodroplets targeted for tumor uptake will allow selective 
ablation of such tumors without need to otherwise identify the treatment location, keeping the selectivity of targeting the tumor despite the larger focal zones. Additionally, we hypothesize that lower frequency will decrease the cavitation threshold using nanodroplets while facilitating greater bubble expansion compared to higher frequency. This hypothesis is based on previous work showing that lower frequency decreases the histotripsy intrinsic threshold (without droplets) and increases bubble expansion (Vlaisavljevich et al. 2015a; Vlaisavljevich et al. 2015b). While previous work studying acoustic droplet vaporization (ADV) has shown the ADV threshold decreases with increasing frequency due to superharmonic focusing, these effects are expected to be negligible for the frequency range $(345 \mathrm{kHz}-3 \mathrm{MHz})$ and droplet size $(<500 \mathrm{~nm}$ in diameter) used in this study ( $\mathrm{Li}$ et al. 2014; Shpak et al. 2014). Therefore, we hypothesize that lower frequency will reduce the NMH threshold for the conditions used in this work. In this study, we tested this hypothesis by exposing tissue phantoms with and without nanodroplets to histotripsy pulses produced by $345 \mathrm{kHz}, 500 \mathrm{kHz}, 1.5 \mathrm{MHz}$, and $3 \mathrm{MHz}$ histotripsy transducers. First, the probability of generating inertial cavitation from a single 1-2 cycle histotripsy pulse was measured for each frequency, with the cavitation threshold defined as the peak negative pressure at which the probability of generating cavitation, $p_{c a v}$, from a single histotripsy pulse was 0.5 (i.e. $p_{c a v}=0.5$ ). Next, the effect of frequency on the size of NMH bubbles was compared using high speed optical imaging. Finally, the ability of PFP-encapsulated nanodroplets to act as sustainable cavitation nuclei over multiple pulses was investigated, as multiple pulses (often $>50$ pulses) are needed to completely destroy all the cells within the treatment volume. Overall, these results will improve our understanding of the $\mathrm{NMH}$ process and help guide parameter optimization for multi-focal tumor ablation using NMH. 


\section{Methods}

\section{Formulation and Characterization of Nanodroplets}

The ideal polymer composition and PFP content of the nanodroplets used in this study were identified in our previous study (Yuksel Durmaz et al. 2014). A poly(ethylene glycol)- $b$ poly(acrylic acid)- $b$-poly(heptadecafluorodecyl methacrylate-co-methyl methacrylate) triblock copolymer was synthesized using a combination of atom transfer radical polymerization (ATRP) and "click" coupling techniques to prepare PFP-loaded nanodroplets (Yuksel Durmaz et al. 2014). Briefly, the copolymers were dissolved in tetrahydrofuran anhydrous (THF, >99.9 \%, Sigma-Aldrich, St. Louis, MO, USA) $(0.2 \% \mathrm{w} / \mathrm{v})$ and cooled down to $0^{\circ} \mathrm{C}$ before the addition of perfluoropentane (PFP, 97\% ca. 85\% n-isomer, Alfa Aesar, Ward Hill, Massachusetts, USA) $(2 \% \mathrm{v} / \mathrm{v})$ while vigorously stirring the reaction mixture. An equal amount of water was slowly added to this solution mixture to trigger micelle formation and the mixture was stirred for $1 \mathrm{~h}$ in an ice bath. The micelles solution was transferred into a dialysis bag (MWCO of $1 \mathrm{KDa}$, Spectrum, Rancho Dominguez, CA, US) and dialyzed against ice-cold 2-( $N$-morpholino) ethanesulfonic acid monohydrate solution (MES, $99 \%$, Acros Organics, Geel, Belgium) of pH 5.5 for 12 hours to remove the THF and get a milky solution of non- cross-linked PFP-loaded nanodroplets. This milky solution was transferred to a round bottom flask and mixed with the 2,2`(ethylenedioxy)-bis(ethylamine) cross-linker (98\% 2,2`-(ethylenedioxy)-bis(ethylamine), Sigma-Aldrich, St. Louis, MO, USA) which reacts with the central PAA block in the polymer backbone via NHS/EDC coupling chemistry (97\% N-hydroxy succinimide; 98\% N-(3Dimethylaminopropyl)-N'-ethylcarbodiimide hydrochloride, Sigma-Aldrich, St. Louis, MO, USA) forming cross-linked nanodroplets with a flexible polymer shell. Shell cross-linked 
nanodroplets were dialyzed against ice-cold water for 12 hours to remove the byproducts of the cross-linkage reaction.

Concentration and size distribution of the nanodroplets were measured using Nanoparticle Tracking Analysis (NTA). Briefly, the NanoSight ${ }^{\mathrm{TM}}$ LM10 (Malvern Instruments, Amesbury, UK), equipped with a temperature-controlled $405 \mathrm{~nm}$ laser module, high sensitivity Scientific Complementary Metal-Oxide-Semiconductor (sCMOS) camera (Hamamatsu, Orca, Hamamatsu City, Japan), and a syringe pump was used for the collection of NTA data. Upon diluting the nanodroplet solution to the appropriate particle concentration with deionized (DI) water (Thermo Scientific, GenPure, Waltham, MA, US), image capture and analysis was carried out using the NTA software (Version 3.0, Build 0066, Malvern Instruments, Amesbury, UK) at $37^{\circ} \mathrm{C}$. The samples were measured by capturing 60 s videos ( 5 videos per each sample). Figure $\mathbf{1}$ is a representative plot showing the size distribution for a single sample of nanodroplets. The error bars represent the standard deviation of the repeat measurements of each sample. The mean size and standard deviation values obtained by the NTA software correspond to arithmetic values calculated with the sizes of all particles analyzed for each sample $(n=5)$. Results from all samples demonstrated that the average size of the nanodroplets (NDs) was $177.9 \pm 1.9 \mathrm{~nm}$ with $10 \%$ of NDs have a diameter $\leq 111 \pm 1.4 \mathrm{~nm}, 50 \%$ of the NDs have a diameter $\leq 155.7 \pm 2.1 \mathrm{~nm}, 90 \%$ of the NDs have a diameter $\leq 268.5 \pm 6.7 \mathrm{~nm}$, and $>99 \%$ of the NDs smaller than $400 \mathrm{~nm}$ and $<0.01 \%$ larger than $600 \mathrm{~nm}$. These results clearly show that the average size of the NDs is much smaller than the size cutoff $(\sim 500 \mathrm{~nm})$ of the tumor vasculature (Gao et al. 2008; Sheeran et al. 2011).

\section{Preparation of Tissue Phantoms}


Agarose phantoms were used to provide a well-controlled viscoelastic medium for this study, as histotripsy bubble behavior is highly dependent on the tissue mechanical properties (Vlaisavljevich et al. 2014a; Vlaisavljevich et al. 2014b; Vlaisavljevich et al. 2014c). The Young's modulus of the agarose tissue phantom was $38 \mathrm{kPa}$, which is within the range of the Young's modulus of hepatocellular carcinoma tumors (20.4-75 kPa), metastatic liver tumors (23.6-75 kPa), and prostate tumors (24 kPa) (Normand et al. 2000; Masuzaki et al. 2007; Zhang et al. 2008). Tissue phantoms containing $1 \%$ agarose w/v were prepared by slowly mixing agarose powder (Agarose Type VII; Sigma-Aldrich, St. Louis, MO, USA) into saline solution ( $0.9 \%$ sodium chloride; Hospira, Lake Forest, Illinois, USA) heated to boiling temperature. The solution was stirred on a hot plate until the gel turned completely transparent and then allowed to boil for ten minutes. After boiling, solutions were allowed to cool and were degassed under a partial vacuum ( $20 \mathrm{kPa}$, absolute) for 30 minutes. After degassing, phantoms containing nanodroplets were prepared by slowly adding the nanodroplets $\left(2.0 \times 10^{8}\right.$ particles $\left./ \mathrm{mL}\right)$ into the agarose solution while stirring. The agarose mixtures were poured into rectangular polycarbonate holders with acoustic windows and placed in a refrigerator at $4^{\circ} \mathrm{C}$ to allow the solution to solidify, forming tissue phantoms with embedded nanodroplets (test) or without nanodroplets

(control). A nanodroplet concentration of $2.0 \times 10^{8}$ particles $/ \mathrm{mL}$ was used for all samples, as preliminary experiments demonstrated that lower concentrations (i.e. $\sim 10^{6}-10^{7}$ particles $/ \mathrm{mL}$ ) did not significantly reduce the cavitation threshold in comparison to control conditions.

\section{Histotripsy Pulse Generation}

Histotripsy pulses were generated at four ultrasound frequencies $(345 \mathrm{kHz}, 500 \mathrm{kHz}, 1.5$ $\mathrm{MHz}$, and $3 \mathrm{MHz}$ ) using three custom-built histotripsy transducers. The $345 \mathrm{kHz}$ pulses were 
generated by a twenty-element array transducer with a geometric focus of $150 \mathrm{~mm}$, an aperture size of $272 \mathrm{~mm}$, and an effective f-number of 0.55 . The $1.5 \mathrm{MHz}$ pulses were generated by a sixelement array transducer with a geometric focus of $55 \mathrm{~mm}$, an aperture of $79 \mathrm{~mm}$ in the elevational direction and $69 \mathrm{~mm}$ in the lateral direction, and effective f-numbers of 0.7 and 0.8 in the elevational and lateral directions, respectively. The $500 \mathrm{kHz}$ and $3 \mathrm{MHz}$ pulses were generated by a dual frequency array transducer that consisted of twelve $500-\mathrm{kHz}$ elements and seven 3-MHz elements. For the $500 \mathrm{kHz}$ elements, the geometric focus was $40 \mathrm{~mm}$, the aperture size was $71 \mathrm{~mm}$, and the effective f-number was 0.56 . For the $3 \mathrm{MHz}$ elements, the geometric focus was $40 \mathrm{~mm}$, the aperture size was $80 \mathrm{~mm}$, and the effective f-number was 0.5 . The design of this dual frequency transducer has been described in detail in a previous study (Lin et al. 2014).

To compare the NMH cavitation threshold with the histotripsy intrinsic threshold, short pulses with a single dominant negative pressure half-cycle were applied to the tissue phantoms with and without nanodroplets. To generate a short therapy pulse, a custom high-voltage pulser developed in-house was used to drive the transducers. The pulser was connected to a fieldprogrammable gate array (FPGA) board (Altera DE1 Terasic Technology, Dover, DE, USA) specifically programmed for histotripsy therapy pulsing. This setup allowed the transducers to output short pulses of less than two cycles. A fiber-optic probe hydrophone built in-house (Parsons et al. 2006b) was used to measure the acoustic output pressure of the transducers. At higher pressure levels ( $p$ - > $23 \mathrm{MPa}$ ), the acoustic output could not be directly measured due to cavitation at the fiber tip. These pressures were estimated by a summation of the output focal peak negative pressure $(p-)$ values from individual transducer elements. This approximation assumes minimal nonlinear distortion of the waveform occurs within the focal region. In a 
previous study (Maxwell et al. 2013), this estimated p-was found to be accurate within $15 \%$ compared to direct focal pressure measurements in water and in a medium (1,3 butanediol) with a higher cavitation threshold. Sample acoustic waveforms produced by the four frequency transducers are shown in Figure 2.

\section{Optical Imaging and Image Processing}

High speed optical imaging was used to capture images of the focal zone after the propagation of each pulse through the focus for water and agarose tissue phantoms using two high speed cameras (Fig. 3). The optics were varied for the different transducers to provide the highest resolution based on the geometric constraints of the transducers and the available optical windows. For experiments with the $345 \mathrm{kHz}$ and $1.5 \mathrm{MHz}$ transducers, a high-speed, 1 megapixel CCD camera (Phantom V210, Vision Research, Wayne, NJ, USA) was aligned with the transducer and backlit by a continuous white-light source. The camera was focused using a macro-bellows lens (Tominon 1:4.5, F=105 mm; Kyocera, Kyoto, Japan), giving the captured images a resolution of approximately $5.9 \mu \mathrm{m}$ per pixel and $3.4 \mu \mathrm{m}$ per pixel for $345 \mathrm{kHz}$ and 1.5 $\mathrm{MHz}$, respectively. For experiments with the $500 \mathrm{kHz}$ and $3 \mathrm{MHz}$ dual frequency transducer, a digital, 1.3-megapixel CCD camera (PN: FL3-U3-13Y3M-C, Flea® 3, PointGrey, Richmond, BC, Canada) was positioned perpendicularly to the dual-frequency array transducer facing one of the transducer's optical windows. A Nikon $4 \mathrm{X}$ objective was attached to the camera with extension tubes to magnify the image plane, giving the captured images a resolution of approximately $2.5 \mu \mathrm{m}$ per pixel. A pulsed white-light LED was placed on the diametricallyopposed optical window of the dual-frequency array transducer, which provided back-lit illumination. The cameras were triggered to record one image for each applied pulse. After 
acquisition, shadowgraph images were converted from grayscale to binary by an intensity threshold determined by the background intensity using image processing software (MATLAB, The Mathworks, Natick, MA, USA), as described in a previous study (Maxwell et al. 2013). Bubbles were indicated as any black regions greater than 5 pixels in diameter. By this criterion, the minimum resolvable bubble radius was $14.75 \mu \mathrm{m}, 6.25 \mu \mathrm{m}, 8.5 \mu \mathrm{m}$, and $6.25 \mu \mathrm{m}$ for the 345 $\mathrm{kHz}, 500 \mathrm{kHz}, 1.5 \mathrm{MHz}$, and $3 \mathrm{MHz}$ transducers, respectively.

\section{Passive Cavitation Detection (PCD)}

In addition to high speed imaging, an acoustic method was used to identify cavitation in the focal zone for cavitation threshold experiments. For each experiment, one of the transducer's therapy elements was also used for passive cavitation detection (PCD) to detect the presence of cavitation in the focal region (Fig. 3). The PCD signal was connected to an oscilloscope (LT372; Lecroy, Chestnut Ridge, NY, USA) with the time window selected to record the backscattering of the therapy pulse from cavitation bubbles (Maxwell et al. 2013; Vlaisavljevich et al. 2014b; Vlaisavljevich et al. 2015a). To determine whether cavitation occurred during a pulse, the signal generated by backscattering of the incident pulse from the focus was analyzed following the method used in previous studies (Maxwell et al. 2013; Vlaisavljevich et al. 2015a). A significant fraction of the incident wave energy is scattered when a cavitation bubble expands, greatly increasing the backscattered pressure amplitude received by the PCD. This signal appeared on the PCD at the time point corresponding to two times the time of flight for the focal length of the respective transducers. The integrated frequency power spectrum $\left(S_{P C D}\right)$ of the backscatter signal was used as a measure of whether cavitation occurred according to the method previously described by Maxwell et al (Maxwell et al. 2013). 


\section{NMH Cavitation Threshold}

For cavitation threshold experiments, 100 pulses were applied inside each sample at each pressure level at a pulse repetition frequency (PRF) of $0.5 \mathrm{~Hz}$. The PRF was kept low to minimize the possibility that cavitation from one pulse would change the probability of cavitation on a subsequent pulse. In a previous study, it was demonstrated that cavitation during a pulse increased the likelihood of cavitation on a following pulse for PRFs $>1 \mathrm{~Hz}$, but this effect was not observed for PRFs below $1 \mathrm{~Hz}$ (Maxwell et al. 2013). In addition to this low PRF, the focus was translated for each pulse by $1 \mathrm{~mm}$ transverse to the acoustic propagation direction in a $10 \times 10$ grid in order to minimize the effects of cavitation damage to the nanodroplets or tissue phantom sample from altering the probability of cavitation. For each pulse, cavitation was monitored using both high speed imaging and PCD, and the fraction of total pulses (out of 100) for which cavitation was detected was determined as the cavitation probability.

The probability of observing cavitation followed a sigmoid function, given by

$$
P\left(p_{-}\right)=\frac{1}{2}+\operatorname{erf}\left(\frac{p_{-}-p_{t}}{\sqrt{2 \sigma^{2}}}\right)
$$

where $\operatorname{erf}$ is the error function, $p_{t}$ is the negative pressure at which the probability $p_{c a v}=0.5, \sigma$ is a variable related to the width of the transition between $p_{c a v}=0$ and $p_{c a v}=1$, with $\pm \sigma$ giving the difference in pressure from about $p_{c a v}=0.15$ to $p_{c a v}=0.85$ for the fit (Maxwell et al. 2013). The cavitation threshold for each sample, $p_{t}$, is defined as the $p$ - corresponding to $p_{c a v}=0.5$ as calculated by the curve fit. Curve fitting for all data sets was performed using an OriginLab curve fitting program (OriginPro 9.1; OriginLab Corporation, Northampton, MA, USA). The fit curves for all samples were analyzed statistically to determine whether the differences in the values of $p_{t}$ were significantly different from each other. The standard errors for $p_{t}$ were 
estimated by a covariance matrix using the delta method (Hosmer and Lemeshow 1992). The

curves were compared using a two-sample t-test with statistic $t\left(p_{\text {int } 1}-p_{\text {int } 2}, \sqrt{S E_{1}^{2}+S E_{2}^{2}}\right)$ at a $95 \%$ confidence interval. Results were considered statistically significant for $p<0.05$. Note that the standard error does not include the uncertainty in absolute pressure from the hydrophone measurement, only the uncertainty in the fit, because the values $p_{t}$ are relative. A sample size of 3 tissue phantoms was used for each experimental condition.

\section{NMH Bubble Size}

To determine if nanodroplet-mediated cavitation bubbles were expanded similarly to histotripsy bubbles at higher pressure, optical images of the growth and collapse of bubbles were recorded by the high-speed camera. Nanodroplet-mediated cavitation bubbles were generated by the $345 \mathrm{kHz}, 500 \mathrm{kHz}, 1.5 \mathrm{MHz}$, and $3 \mathrm{MHz}$ transducers at peak negative pressures slightly above the nanodroplet cavitation threshold at each frequency. More specifically, the peak negative pressure was $10.8 \mathrm{MPa}(345 \mathrm{kHz}), 10.3 \mathrm{MPa}(500 \mathrm{kHz}), 12.9 \mathrm{MPa}(1.5 \mathrm{MHz})$, and 14.7 $\mathrm{MPa}(3 \mathrm{MHz})$. For comparison, bubbles were produced in tissue phantoms without nanodroplets at estimated peak negative pressures of $26.4 \mathrm{MPa}(345 \mathrm{kHz}), 26.3 \mathrm{MPa}(500 \mathrm{kHz}), 26.8 \mathrm{MPa}$ (1.5 MHz), and 28.8 MPa (3 MHz), which is slightly above the intrinsic threshold without nanodroplets. The maximum radius of bubbles was compared by reconstructing the average expansion and collapse behavior using a series of time-delayed images of the bubbles produced by identical histotripsy pulses. The specific delay times were varied based on the frequency and sample in order to reconstruct a sequence of bubble images and determine the time point corresponding to the maximum bubble radius, $R_{\max }$. The $R_{\max }$ was compared between samples by analyzing results from 20 identical histotripsy pulses recorded at the time of maximum 
expansion, with each pulse applied to a different point in the sample ( $2 \mathrm{~mm}$ spacing) to prevent the effects of cavitation damage from altering the tissue phantom and nanodroplet properties. The size of single bubbles was measured for all twenty pulses to determine the bubble radius, and the mean and standard deviation in bubble radius were calculated. A sample size of 6 tissue phantoms was used for each experimental condition.

\section{NMH Multi-pulse Sustainability}

To determine if nanodroplets are sustainable cavitation nuclei over multiple pulses, 1000 ultrasound pulses were applied to a single focal region in tissue phantoms containing nanodroplets at a PRF of $1 \mathrm{~Hz}$ and peak negative pressures of $10.8 \mathrm{MPa}(345 \mathrm{kHz}), 10.3 \mathrm{MPa}$ (500 kHz), 12.9 MPa (1.5 MHz), and 14.7 MPa (3 MHz). In a previous study, nanodropletmediated histotripsy created consistent, well-defined fractionation in tissue phantoms at $10 \mathrm{~Hz}$ PRF by maintaining cavitation at over multiple pulses (Vlaisavljevich et al. 2013a). However, it is unclear whether the nanodroplets themselves or the residual nuclei from previous pulses are responsible for seeding cavitation after the first few pulses. As a result, the PRF in this study was kept low $(1 \mathrm{~Hz})$ to minimize the contributions of residual nuclei from a previous pulse from effecting cavitation generation on a subsequent pulse in order to determine if nanodroplets continue to function as viable cavitation nuclei after the first few pulses or if the nanodroplets are destroyed in the cavitation process. Cavitation was monitored using high speed optical imaging, and the number of bubbles produced by each pulse was compared for 1000 histotripsy pulses in each sample. In order to quantify the ability of nanodroplets to sustain a cavitation bubble cloud over multiple pulses, the number of pulses before cloud extinction, $P \#_{E x t}$, was plotted as a 
function of frequency. A sample size of 6 tissue phantoms was used for each experimental condition.

\section{Results}

\section{NMH Cavitation Threshold}

To investigate the effects of ultrasound frequency on the NMH threshold, histotripsy pulses were applied to tissue-mimicking agarose phantoms with and without nanodroplets using the $345 \mathrm{kHz}, 500 \mathrm{kHz}, 1.5 \mathrm{MHz}$, and $3 \mathrm{MHz}$ histotripsy transducers. For all frequencies, cavitation bubbles were observed on the high-speed camera when a certain negative pressure was exceeded, with close agreement between optical imaging and PCD detection methods (Fig. 4), as seen in previous studies (Maxwell et al. 2013; Vlaisavljevich et al. 2015a). Results from phantoms without nanodroplets show that the histotripsy intrinsic threshold, $p_{t_{-} i n t}$, was $p_{t_{-} i n t}$ $=24.8 \pm 1.1 \mathrm{MPa}$, with $\sigma_{\text {mean }}=2.0 \mathrm{MPa}$ for $345 \mathrm{kHz} ; p_{t_{-} \text {int }}=25.5 \pm 1.7 \mathrm{MPa}$, with $\sigma_{\text {mean }}=1.8 \mathrm{MPa}$ for $500 \mathrm{kHz} ; p_{t_{-} \text {int }}=26.7 \pm 0.4 \mathrm{MPa}$, with $\sigma_{\text {mean }}=1.0 \mathrm{MPa}$ for $1.5 \mathrm{MHz}$; and $p_{t_{-} \text {int }}=26.8 \pm 0.5 \mathrm{MPa}$, with $\sigma_{\text {mean }}=0.9 \mathrm{MPa}$ for $3 \mathrm{MHz}$ (Fig. 5). Note that at lower amplitudes, cavitation was occasionally observed that deviated from the curve function, especially at lower frequency. These cavitation events were probably caused by contamination of the sample by heterogeneities in the liquid that could not be entirely avoided throughout the experiment. The effects of nanodroplets on the cavitation threshold demonstrated a significant decrease in the cavitation threshold compared to the histotripsy intrinsic threshold (Fig. 5). The NMH threshold for phantoms containing PFP nanodroplets, $p_{t_{-} P F P}$, was $p_{t_{-} P F P}=7.4 \pm 0.1 \mathrm{MPa}$, with $\sigma_{\text {mean }}=1.4 \mathrm{MPa}$ for $345 \mathrm{kHz} ; p_{t_{-} P F P}=9.2 \pm 0.9 \mathrm{MPa}$, with $\sigma_{\text {mean }}=0.8 \mathrm{MPa}$ for $500 \mathrm{kHz} ; p_{t_{-} P F P}=10.5 \pm 0.2 \mathrm{MPa}$, with $\sigma_{\text {mean }}=0.4 \mathrm{MPa}$ for $1.5 \mathrm{MHz}$; and $p_{t_{-} P F P}=13.2 \pm 0.4 \mathrm{MPa}$, with $\sigma_{\text {mean }}=0.6 \mathrm{MPa}$ for $3 \mathrm{MHz}$ 
(Fig. 5). The results of the cavitation threshold for all frequencies are plotted in Figure 6. Comparing the results for the NMH threshold and histotripsy intrinsic threshold demonstrated a significant decrease in the cavitation threshold and a significant increase in the steepness of the S-curve $(\sigma)$ for samples containing nanodroplets at all frequencies. Additionally, it was observed that the NMH threshold was significantly decreased at lower frequency (Fig.6).

\section{NMH Bubble Size}

To study the effects of ultrasound frequency on the expansion of NMH bubbles, the maximum bubble radius, $\mathrm{R}_{\max }$, produced by $345 \mathrm{kHz}, 500 \mathrm{kHz}, 1.5 \mathrm{MHz}$, and $3 \mathrm{MHz}$ histotripsy pulses was measured in phantoms with and without nanodroplets. The peak negative pressure used for each frequency was chosen to be slightly above the cavitation threshold at each frequency so that cavitation was always generated $\left(\mathrm{P}_{\mathrm{cav}}=1\right)$. Optical imaging results demonstrated that $\mathrm{NMH}$ bubbles were significantly smaller than histotripsy bubbles generated above the intrinsic threshold (Fig.7). Note that different image magnifications were used for each frequency for the images shown in Figure 7, to provide a better image of the bubbles produced at each frequency. At all frequencies, results showed that the $\mathrm{R}_{\max }$ for $\mathrm{NMH}$ bubbles was between $30 \%-40 \%$ of the $\mathrm{R}_{\max }$ measured for histotripsy bubbles produced above the intrinsic threshold (Fig.8). Results further showed that larger bubbles were observed at lower frequency for both the histotripsy only and NMH conditions (Fig.8). For example, $\mathrm{R}_{\max }$ for $\mathrm{NMH}$ bubbles was shown to decrease from $126.7 \pm 47.5 \mu \mathrm{m}$ at $345 \mathrm{kHz}$ to $106.5 \pm 17.6 \mu \mathrm{m}, 34.7 \pm 13.4 \mu \mathrm{m}$, and 12.9 $\pm 5.3 \mu \mathrm{m}$ at $500 \mathrm{kHz}, 1.5 \mathrm{MHz}$, and $3 \mathrm{MHz}$, respectively (Table 1). Comparing $\mathrm{R}_{\max }$ for $\mathrm{NMH}$ and histotripsy bubbles showed that, although $\mathrm{NMH}$ bubbles were significantly smaller than histotripsy bubbles for all frequencies, $\mathrm{NMH}$ bubbles produced at the lower two frequencies 
$(345 \mathrm{kHz}, 500 \mathrm{kHz})$ grew larger than histotripsy bubbles produced at the two higher frequencies

(1.5 MHz, $3 \mathrm{MHz}$ ). For example, the $\mathrm{R}_{\max }$ of $\mathrm{NMH}$ bubbles produced at $345 \mathrm{kHz}$ and $500 \mathrm{kHz}$ $(126.7 \pm 47.5 \mu \mathrm{m}$ and $106.5 \pm 17.6 \mu \mathrm{m})$ were significantly larger than the $R_{\max }$ of histotripsy bubbles generated above the intrinsic threshold at $1.5 \mathrm{MHz}$ and $3 \mathrm{MHz}(79.5 \pm 11.5 \mu \mathrm{m}$ and 34.3 \pm 8.5 ) (Fig.8). The complete list of $\mathbf{R}_{\max }$ results are listed in Table $\mathbf{1}$ along with the peak negative pressure applied for each condition.

\section{NMH Multi-pulse Sustainability}

To determine if nanodroplets are sustainable cavitation nuclei over multiple pulses, 1000 histotripsy pulses were applied to a single focal region in phantoms containing nanodroplets at a PRF of $1 \mathrm{~Hz}$. Results demonstrated that a bubble cloud consisting of many bubbles was observed after the first pulse (Fig.9). However, the number of bubbles observed inside the cloud significantly decreased with increasing number of pulses (Fig.9). For example, for the $3 \mathrm{MHz}$ sample shown in Figure 9, only one bubble was observed after 5 pulses with no bubbles remaining after 10 pulses. At lower frequency, bubbles were generated for more pulses than at higher frequency, but still showed the same trend of decreasing number of bubbles with increasing pulse number (Fig.9). It is likely that bubbles lasted for more pulses at lower frequency due to enhanced bubble expansion resulting in a larger population of residual nuclei and a corresponding increase in dissolution time. Figure $\mathbf{1 0}$ shows the number of pulses before cloud extinction, $P \#_{E x x}$, as a function of frequency $(\mathrm{n}=6)$. Results demonstrated a significant decrease $(\mathrm{p}<0.05)$ in $P \#_{E x t}$ at higher frequencies, with $P \#_{E x t}$ observed to decrease from $80.5 \pm 10.3$ pulses at $345 \mathrm{kHz}$ to $51.7 \pm 7.3$ pulses, $15.7 \pm 4.8$ pulses, and $5.5 \pm 1.9$ pulses at $500 \mathrm{kHz}, 1.5 \mathrm{MHz}$, 
and $3 \mathrm{MHz}$, respectively (Fig.10). For all frequencies, no bubbles were observed after 100 pulses were applied to the samples.

\section{Discussion}

In this work, we investigated the effects of frequency on nanodroplet-mediated histotripsy (NMH) using our polymer encapsulated PFP nanodroplets (Yuksel Durmaz et al. 2014). This work builds upon two previous studies which demonstrated that NMH has the potential to be used for targeted tissue ablation by decreasing the pressure threshold required to generate histotripsy bubbles (Vlaisavljevich et al. 2013a; Yuksel Durmaz et al. 2014). In this study, we investigated the effects of frequency in order to optimize the acoustic parameters used for NMH therapy. Since one of the goals of NMH therapy is to efficiently treat large and/or multi-focal tumor nodules, $\mathrm{NMH}$ parameters should be optimized to increase the size of the focal zone, decrease the NMH cavitation threshold, and increase NMH bubble expansion, all of which were improved at lower frequencies as shown in this study.

In the first part of this study, the effects of frequency on the NMH cavitation threshold were investigated, with results demonstrating that PFP nanodroplets significantly reduced the cavitation threshold compared to the histotripsy intrinsic threshold. At all frequencies tested, the NMH threshold was significantly lower than the histotripsy intrinsic threshold, while maintaining a steep threshold behavior. This distinct threshold behavior is promising for the development of NMH therapy, in which the applied pressure must be chosen in the region above the NMH threshold but below the histotripsy intrinsic threshold to ensure cavitation is only generated in regions containing nanodroplets. The results of this study show that all the frequencies investigated $(345 \mathrm{kHz}-3 \mathrm{MHz})$ could be used for $\mathrm{NMH}$, with lower frequencies 
offering the largest drop in threshold compared to the histotripsy intrinsic threshold while maintaining the distinct threshold behavior. These results suggest that lower frequencies will be more efficient for NMH applications in which large volume or multi-focal ablation is desired. However, it should be noted that lower frequencies may reduce the treatment precision due to the larger focal volume, which would increase the likelihood of generating collateral damage outside of the desired treatment region. As such, higher frequencies may be preferred for NMH applications in which very high precision is required.

The frequency dependence of the NMH cavitation threshold observed in this study is opposite to that reported in previous work on acoustic droplet vaporization (ADV) which showed that the ADV threshold decreases with increasing frequency (Kripfgans et al. 2000; Schad and Hynynen 2010; Williams et al. 2013). However, those studies used larger droplets, higher frequency ranges, and/or longer duration exposures. Recent work has revealed that the decrease in the ADV threshold at higher frequencies is due to superharmonic focusing inside the droplet, which is significantly enhanced at higher frequencies and in larger droplets ( $\mathrm{Li}$ et al. 2014; Shpak et al. 2014). These previous studies help to explain why the cavitation thresholds in this study do not follow the trends observed for ADV, since the extent of superharmonic focusing is negligible for the droplet size $(<400 \mathrm{~nm})$ and frequencies $(\leq 3 \mathrm{MHz})$ used in this study (Shpak et al. 2014). In contrast to those previous studies, the results of this work suggest that NMH bubbles are generated inside the droplets directly from the incident $p$ - (tensile portion of the incident wave), similar to histotripsy bubbles generated above the intrinsic threshold (Vlaisavljevich et al. 2015a). This hypothesis is supported by the trends in the cavitation threshold with lower frequencies resulting in a lower cavitation threshold, likely due to the longer duration of the applied $p$ - and the larger focal zone at lower frequencies, which increases the volume of PFP 
exposed to the $p$ - and therefore increases the probability of nucleating cavitation inside the PFP nanodroplets. This would also imply that the NMH threshold will be affected by nanodroplet concentration, since a higher concentration of nanodroplets would result in a larger volume of PFP being exposed to the applied $p$-. This would explain why a concentration of $2.0 \times 10^{8}$ particles $/ \mathrm{mL}$ was required in order to reduce the cavitation threshold in this study while concentrations $<\sim 10^{6}-10^{7}$ particles $/ \mathrm{mL}$ did not significantly reduce the cavitation threshold compared to the histotripsy intrinsic threshold. This threshold behavior suggests that the nanodroplets decrease the cavitation threshold by carrying a lower threshold media (PFP) rather than by acting as discrete nuclei as would be the case for gas-filled contrast agents. Future work will further investigate the role of droplet concentration on NMH therapy.

In the second part of this study, the effects of frequency on NMH bubble expansion were investigated, with results showing that lower frequencies facilitated larger bubble expansion. While it is likely that NMH will be able to fractionate tissue even at higher frequencies, the results of this study suggest that $\mathrm{NMH}$ will be more efficient at lower frequencies due to enhanced bubble expansion. A previous study investigating the effects of frequency on histotripsy bubble expansion demonstrated that the increase in bubble expansion at lower frequencies is due the increased duration of the applied rarefactional pressure (Vlaisavljevich et al. 2015b). This same effect likely explains the results of this study in which NMH bubbles at lower frequencies grew larger than bubbles produced by histotripsy without nanodroplets at higher frequencies, despite a $>18 \mathrm{MPa}$ decrease in the peak negative pressure of the applied pulses. For example, NMH bubbles produced at $345 \mathrm{kHz}$ and $500 \mathrm{kHz}$ grew larger than bubbles produced by histotripsy without nanodroplets at $1.5 \mathrm{MHz}$ and $3 \mathrm{MHz}$, which have previously been shown capable of achieving tissue fractionation (Lin et al. 2014; Vlaisavljevich et al. 
2014c). This finding supports our hypothesis that NMH can be used to efficiently create histotripsy lesions when using lower frequencies.

In the final part of this work, the ability of PFP nanodroplets to act as sustainable cavitation nuclei over multiple pulses was investigated. In a previous study, nanodropletmediated histotripsy created consistent, well-defined fractionation at pressure levels (11-20 MPa) significantly below the histotripsy intrinsic threshold ( 26-30 MPa) in tissue phantoms at a PRF of $10 \mathrm{~Hz}$ by maintaining cavitation at over multiple pulses (Vlaisavljevich et al. 2013a). However, it is unclear whether the nanodroplets themselves or residual nuclei from previous pulses were responsible for seeding cavitation after the first few pulses. In this study, the PRF was kept low $(1 \mathrm{~Hz})$ to minimize the contributions of residual nuclei from a previous pulse, with results showing a significant reduction in the number of bubbles generated by $\mathrm{NMH}$ with increasing number of pulses. For all frequencies, no bubbles were observed in tissue phantoms after 100 pulses. These results suggest that the nanodroplets are destroyed by the cavitation process and only function as cavitation nuclei for the first few pulses, potentially due to the PFP being dissolved after undergoing the NMH cavitation process. This finding indicates that NMH will need to be applied at a higher PRF in order sustain cavitation for the duration of the treatment, since previous work has shown that multiple pulses (often $>50$ pulses) are needed to completely destroy all the cells within the treatment volume. It is also possible that nanodroplets containing a higher boiling point perfluorocarbon, such as perfluorohexane, would re-condense into a liquid and remain sustainable nuclei over multiple pulses, which would be a major benefit for NMH therapy, as previous work has shown a decrease in ablation efficiency for higher PRF treatments that rely on residual nuclei from previous pulses to maintain the cavitation bubble cloud (Wang et al. 2012). However, higher boiling point droplets may also require a higher 
pressure in order to generate cavitation, which would not be desired for NMH therapy. Future work will investigate the possibility of using nanodroplets containing a higher boiling point perfluorocarbon for NMH therapy.

\section{Conclusions}

In this work, the effects of ultrasound frequency on nanodroplet-mediated histotripsy were investigated, with results supporting our hypothesis that using a lower frequency will improve NMH therapy. The results demonstrated that the NMH threshold was significantly reduced at lower frequencies, ranging from $7.4 \mathrm{MPa}$ at $345 \mathrm{kHz}$ to $13.2 \mathrm{MPa}$ at $3 \mathrm{MHz}$. Furthermore, the results demonstrated that NMH bubble expansion was enhanced at lower frequency, generating bubbles with a maximum radius $>100 \mu \mathrm{m}$ despite the reduced pressure applied. Finally, multipulse experiments demonstrated that nanodroplets are destroyed during the first few pulses at $1 \mathrm{~Hz}$ PRF. Overall, the results of this study provide significant insight in the role of ultrasound parameters in NMH therapy and will provide a rational basis to specifically tailor acoustic parameters in order to improve NMH tissue fractionation.

\section{Acknowledgements}

We would like to thank Sonja Capracotta, PhD (Technical Specialist, Nano Sight, School of Public Health at the University of Michigan) for her help on NTA size and concentration measurements. This material is based upon work supported by a National Science Foundation Graduate Research Fellowship to Eli Vlaisavljevich. Omer Aydin acknowledges the support of the Turkish Republic the Ministry of National Education Fellowship Program (1416). This work was supported by a grant from the United States Department of Defense (W81XWH-11-PCRP-ID). 
Disclosure notice: Drs. Zhen $\mathrm{Xu}$ and Brian Fowlkes have financial interests and/or other relationship with HistoSonics Inc. 


\section{References}

Gao Z, Kennedy AM, Christensen DA, Rapoport NY. Drug-loaded nano/microbubbles for combining ultrasonography and targeted chemotherapy. Ultrasonics 2008;48:260-70.

Hall TL, Kieran K, Ives K, Fowlkes JB, Cain CA, Roberts WW. Histotripsy of rabbit renal tissue in vivo: temporal histologic trends. J Endourol 2007;21:1159-66.

Hempel CR, Hall TL, Cain CA, Fowlkes JB, Xu Z, Roberts WW. Histotripsy fractionation of prostate tissue: local effects and systemic response in a canine model. J Urol 2011;185:1484-9.

Hosmer DW, Lemeshow S. Confidence interval estimation of interaction. Epidemiology 1992;3:452-6.

Kripfgans OD, Fowlkes JB, Miller DL, Eldevik OP, Carson PL. Acoustic droplet vaporization for therapeutic and diagnostic applications. Ultrasound Med Biol 2000;26:1177-89.

Li DS, Kripfgans OD, Fabiilli ML, Brian Fowlkes J, Bull JL. Initial nucleation site formation due to acoustic droplet vaporization. Appl Phys Lett 2014;104:063703.

Lin KW, Duryea AP, Kim Y, Hall TL, Xu Z, Cain CA. Dual-beam histotripsy: a low-frequency pump enabling a high-frequency probe for precise lesion formation. IEEE Trans Ultrason Ferroelectr Freq Control 2014;61:325-40.

Masuzaki R, Tateishi R, Yoshida H, Sato T, Ohki T, Goto T, Sato S, Sugioka Y, Ikeda H, Shiina $\mathrm{S}$, Kawabe T, Omata M. Assessing liver tumor stiffness by transient elastography. Hepatol Int 2007;1:394-7.

Maxwell AD, Cain CA, Hall TL, Fowlkes JB, Xu Z. Probability of cavitation for single ultrasound pulses applied to tissues and tissue-mimicking materials. Ultrasound Med Biol 2013;39:449-65.

Maxwell AD, Owens G, Gurm HS, Ives K, Myers DD, Jr., Xu Z. Noninvasive treatment of deep venous thrombosis using pulsed ultrasound cavitation therapy (histotripsy) in a porcine model. J Vasc Interv Radiol 2011;22:369-77.

Normand V, Lootens DL, Amici E, Plucknett KP, Aymard P. New insight into agarose gel mechanical properties. Biomacromolecules 2000;1:730-8.

Owens GE, Miller RM, Ensing G, Ives K, Gordon D, Ludomirsky A, Xu Z. Therapeutic ultrasound to noninvasively create intracardiac communications in an intact animal model. Catheter Cardiovasc Interv 2011;77:580-8.

Parsons JE, Cain CA, Abrams GD, Fowlkes JB. Pulsed cavitational ultrasound therapy for controlled tissue homogenization. Ultrasound Med Biol 2006a;32:115-29. 
Parsons JE, Cain CA, Fowlkes JB. Cost-effective assembly of a basic fiber-optic hydrophone for measurement of high-amplitude therapeutic ultrasound fields. J Acoust Soc Am 2006b;119:143240 .

Parsons JE, Cain CA, Fowlkes JB. Spatial variability in acoustic backscatter as an indicator of tissue homogenate production in pulsed cavitational ultrasound therapy. IEEE Trans Ultrason Ferroelectr Freq Control 2007;54:576-90.

Roberts WW, Hall TL, Ives K, Wolf JS, Jr., Fowlkes JB, Cain CA. Pulsed cavitational ultrasound: a noninvasive technology for controlled tissue ablation (histotripsy) in the rabbit kidney. J Urol 2006;175:734-8.

Schad KC, Hynynen K. In vitro characterization of perfluorocarbon droplets for focused ultrasound therapy. Phys Med Biol 2010;55:4933-47.

Sheeran PS, Luois S, Dayton PA, Matsunaga TO. Formulation and Acoustic Studies of a New Phase-Shift Agent for Diagnostic and Therapeutic Ultrasound. Langmuir 2011;27:10412-20.

Shpak O, Verweij M, Vos HJ, de Jong N, Lohse D, Versluis M. Acoustic droplet vaporization is initiated by superharmonic focusing. Proc Natl Acad Sci U S A 2014;111:1697-702.

Styn NR, Wheat JC, Hall TL, Roberts WW. Histotripsy of VX-2 tumor implanted in a renal rabbit model. J Endourol 2010;24:1145-50.

Vlaisavljevich E, Durmaz YY, Maxwell A, Elsayed M, Xu Z. Nanodroplet-mediated histotripsy for image-guided targeted ultrasound cell ablation. Theranostics 2013a;3:851-64.

Vlaisavljevich E, Kim Y, Allen S, Owens G, Pelletier S, Cain C, Ives K, Xu Z. Image-Guided Non-Invasive Ultrasound Liver Ablation using Histotripsy: Feasibility Study in an In Vivo Porcine Model. . Ultrasound Med Biol 2013b;

Vlaisavljevich E, Kim Y, Owens G, Roberts W, Cain C, Xu Z. Effects of tissue mechanical properties on susceptibility to histotripsy-induced tissue damage. Phys Med Biol 2014a;59:25370.

Vlaisavljevich E, Lin KW, Maxwell A, Warnez MT, Mancia L, Singh R, Putnam AJ, Fowlkes B, Johnsen E, Cain C, Xu Z. Effects of Ultrasound Frequency and Tissue Stiffness on the Histotripsy Intrinsic Threshold for Cavitation. Ultrasound Med Biol 2015a;

Vlaisavljevich E, Lin KW, Warnez MT, Singh R, Mancia L, Putnam AJ, Johnsen E, Cain C, Xu Z. Effects of tissue stiffness, ultrasound frequency, and pressure on histotripsy-induced cavitation bubble behavior. Phys Med Biol 2015b;60:2271-92.

Vlaisavljevich E, Maxwell A, Warnez M, Johnsen E, Cain CA, Xu Z. Histotripsy-induced cavitation cloud initiation thresholds in tissues of different mechanical properties. IEEE Trans Ultrason Ferroelectr Freq Control 2014b;61:341-52. 
Vlaisavljevich E, Warnez M, Johnsen E, Singh R, Putnam A, Xu Z. Investigation of the role of tissue stiffness and ultrasound frequency in histotripsy-induced cavitation. . Symposium on Therapeutic Ultrasound 2014c;

Wang TY, Xu Z, Hall TL, Fowlkes JB, Cain CA. An efficient treatment strategy for histotripsy by removing cavitation memory. Ultrasound Med Biol 2012;38:753-66.

Williams R, Wright C, Cherin E, Reznik N, Lee M, Gorelikov I, Foster FS, Matsuura N, Burns PN. Characterization of submicron phase-change perfluorocarbon droplets for extravascular ultrasound imaging of cancer. Ultrasound Med Biol 2013;39:475-89.

$\mathrm{Xu}$ Z, Fowlkes JB, Ludomirsky A, Cain CA. Investigation of intensity thresholds for ultrasound tissue erosion. Ultrasound Med Biol 2005a;31:1673-82.

$\mathrm{Xu}$ Z, Fowlkes JB, Rothman ED, Levin AM, Cain CA. Controlled ultrasound tissue erosion: the role of dynamic interaction between insonation and microbubble activity. J Acoust Soc Am 2005b;117:424-35.

Xu Z, Owens G, Gordon D, Cain C, Ludomirsky A. Noninvasive creation of an atrial septal defect by histotripsy in a canine model. Circulation 2010;121:742-9.

Xu Z, Raghavan M, Hall TL, Chang CW, Mycek MA, Fowlkes JB, Cain CA. High speed imaging of bubble clouds generated in pulsed ultrasound cavitational therapy--histotripsy. IEEE Trans Ultrason Ferroelectr Freq Control 2007;54:2091-101.

Yuksel Durmaz Y, Vlaisavljevich E, Xu Z, ElSayed M. Development of nanodroplets for histotripsy-mediated cell ablation. Mol Pharm 2014;11:3684-95.

Zhang M, Nigwekar P, Castaneda B, Hoyt K, Joseph JV, di Sant'Agnese A, Messing EM, Strang JG, Rubens DJ, Parker KJ. Quantitative characterization of viscoelastic properties of human prostate correlated with histology. Ultrasound Med Biol 2008;34:1033-42. 


\section{Tables.}

Table 1. Bubble $\mathbf{R}_{\max }$ results. Table shows the maximum bubble radius produced in tissue phantoms with and without nanodroplets, along with the peak negative pressure $(p-)$ applied for each condition. The $p$ - for each condition was chosen to be slightly above the nanodropletmediated histotripsy (NMH) and intrinsic cavitation thresholds of the sample at each frequency.

\begin{tabular}{|c|c|c|c|}
\hline Frequency & Sample & P- (MPa) & Max Radius $(\mu \mathrm{m})$ \\
\hline \multicolumn{4}{|l|}{$345 \mathrm{kHz}$} \\
\hline & No Droplets & 28 & $398.8 \pm 102.5$ \\
\hline & Droplets & 10.8 & $126.7 \pm 47.5$ \\
\hline \multicolumn{4}{|l|}{$500 \mathrm{kHz}$} \\
\hline & No Droplets & 28.1 & $297.4 \pm 50.5$ \\
\hline & Droplets & 10.3 & $106.5 \pm 17.6$ \\
\hline \multicolumn{4}{|l|}{$1.5 \mathrm{MHz}$} \\
\hline & No Droplets & 28.9 & $79.5 \pm 11.5$ \\
\hline & Droplets & 12.9 & $34.7 \pm 13.4$ \\
\hline \multicolumn{4}{|l|}{$3 \mathrm{MHz}$} \\
\hline & No Droplets & 30.3 & $34.3 \pm 8.5$ \\
\hline & Droplets & 14.7 & $12.9 \pm 5.3$ \\
\hline
\end{tabular}




\section{Figures.}

Figure 1. Nanodroplet size distribution. Nanodroplet size distribution results show an average nanodroplet size of $177.9 \pm 1.9 \mathrm{~nm}$.

Figure 2. Acoustic waveforms. Example of a 2-cycle histotripsy pulses generated by the 345 $\mathrm{kHz}, 500 \mathrm{kHz}, 1.5 \mathrm{MHz}$, and $3 \mathrm{MHz}$ histotripsy transducers.

Figure 3. Experimental set-up. The focus of the histotripsy transducer was aligned inside tissue phantoms with and without nanodroplets. Cavitation was monitored using high speed optical imaging and passive cavitation detection using one of the therapy elements.

Figure 4. Cavitation detection. Sample passive cavitation detection (PCD) signals (top) and high speed optical images (bottom) used for cavitation detection. Results showed good agreement between the two methods. Representative images shown above are from $345 \mathrm{kHz}$ histotripsy pulses applied to tissue phantoms containing nanodroplets.

Figure 5. Cavitation threshold curves. Probability curves for tissue phantoms with and without nanodroplets. Results showed a significant decrease in the cavitation threshold with nanodroplets compared to controls. Results also showed a significant increase in the nanodroplet cavitation threshold with increasing frequency. 
Figure 6. Cavitation threshold results. Bar plots show the cavitation threshold results for tissue phantoms with and without perfluoropentane (PFP) nanodroplets at all frequencies studied in this work.

Figure 7. Bubble $\mathbf{R}_{\max }$ Images: . Optical images of bubbles formed in tissue phantoms, with and without nanodroplets, by $345 \mathrm{kHz}, 500 \mathrm{kHz}, 1.5 \mathrm{MHz}$, and $3 \mathrm{MHz}$ histotripsy pulses. Results show a significant decrease in bubble size for nanodroplet-mediated histotripsy bubbles compared with control bubbles produced at the same frequency at higher pressure. Results further show a significant decrease in bubble size with increasing frequency. Note: Different image magnifications were used for the above images to better image the bubbles at each frequency.

Figure 8. Bubble $\mathbf{R}_{\max }$ results. Plot shows a comparison of the maximum bubble radius produced in tissue phantoms with and without nanodroplets by $345 \mathrm{kHz}, 500 \mathrm{kHz}, 1.5 \mathrm{MHz}$, and $3 \mathrm{MHz}$ histotripsy pulses. The peak negative pressures ( $p$-) applied for each condition are listed in Table 1.

Figure 9. Bubble cloud multi-pulse images. Optical images show NMH bubbles produced by $345 \mathrm{kHz}(p-=10.8 \mathrm{MPa})$ and $3 \mathrm{MHz}(p-=14.7 \mathrm{MPa})$ at a single focal point in tissue phantoms containing nanodroplets at a pulse repetition frequency (PRF) of $1 \mathrm{~Hz}$. Results showed a decrease in the number of bubbles observed at the focus with increasing pulse number. 
Figure 10. Bubble cloud multi-pulse sustainability. Plot shows the number of pulses before cloud extinction, $P \#_{E x t}$, as a function of frequency. Results demonstrated a significant decrease $(\mathrm{p}<0.05)$ in $P \#_{E x t}$ with increasing frequency. A sample size of 6 tissue phantoms was used for each experimental condition. 


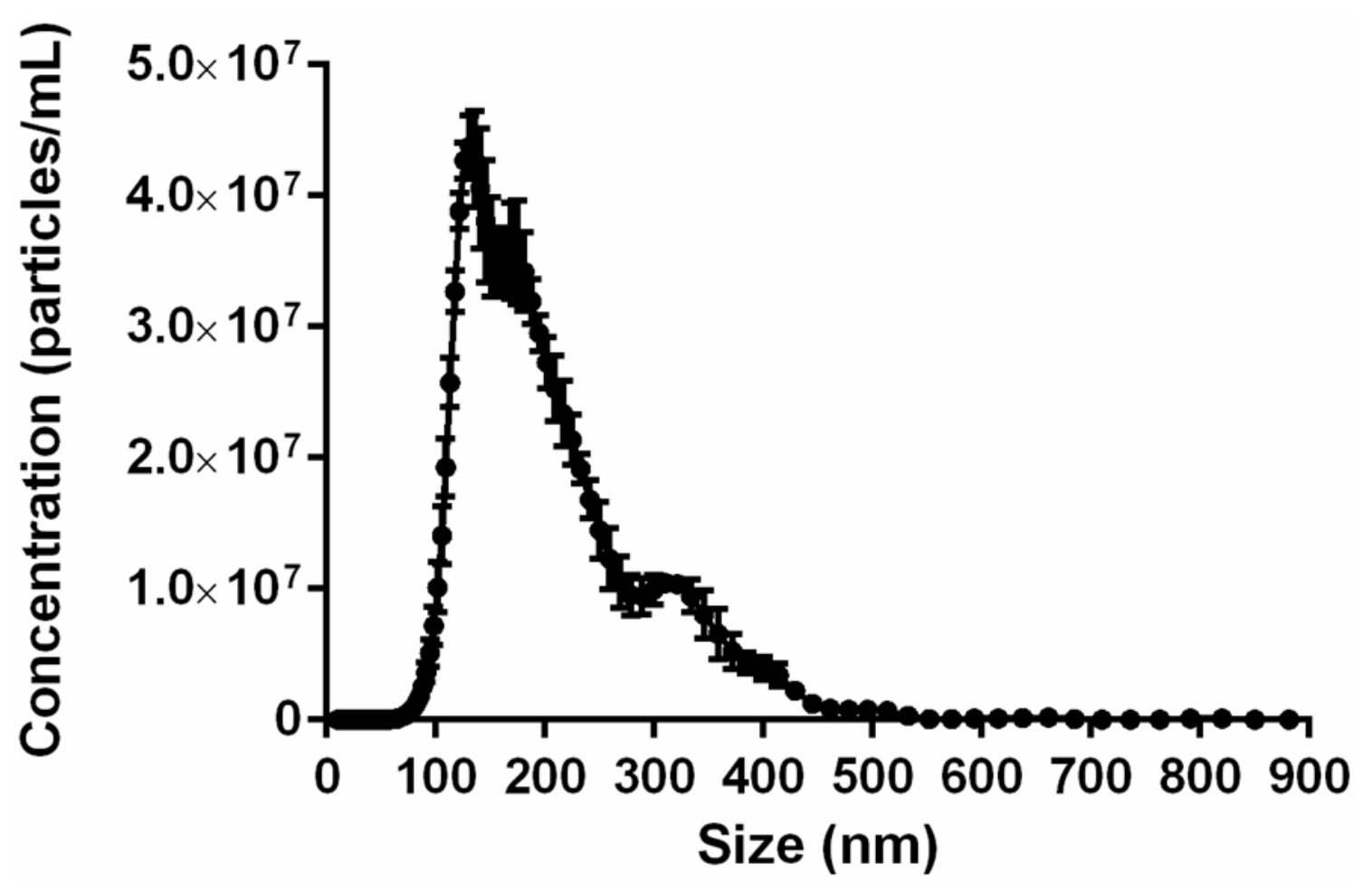


Figure 2
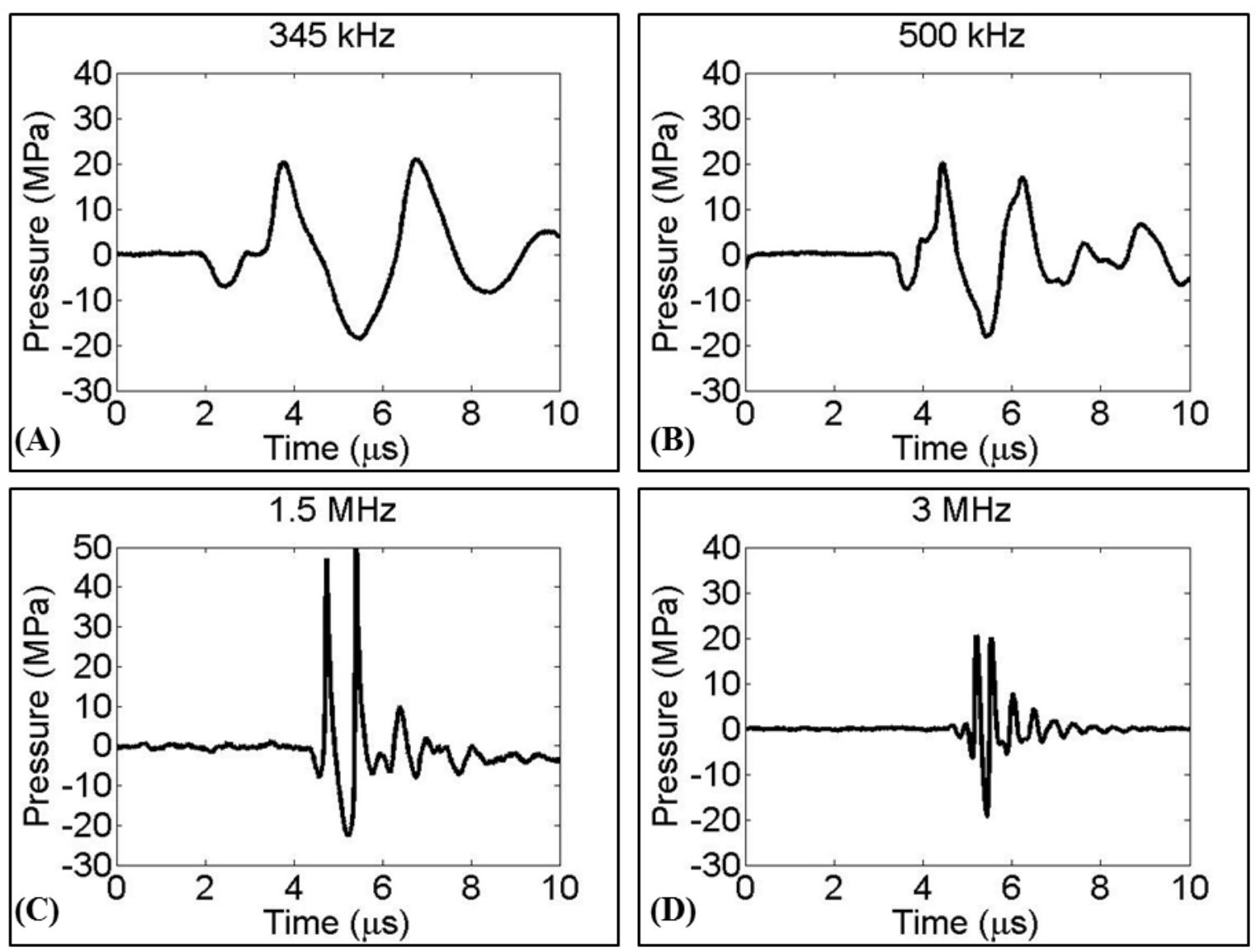


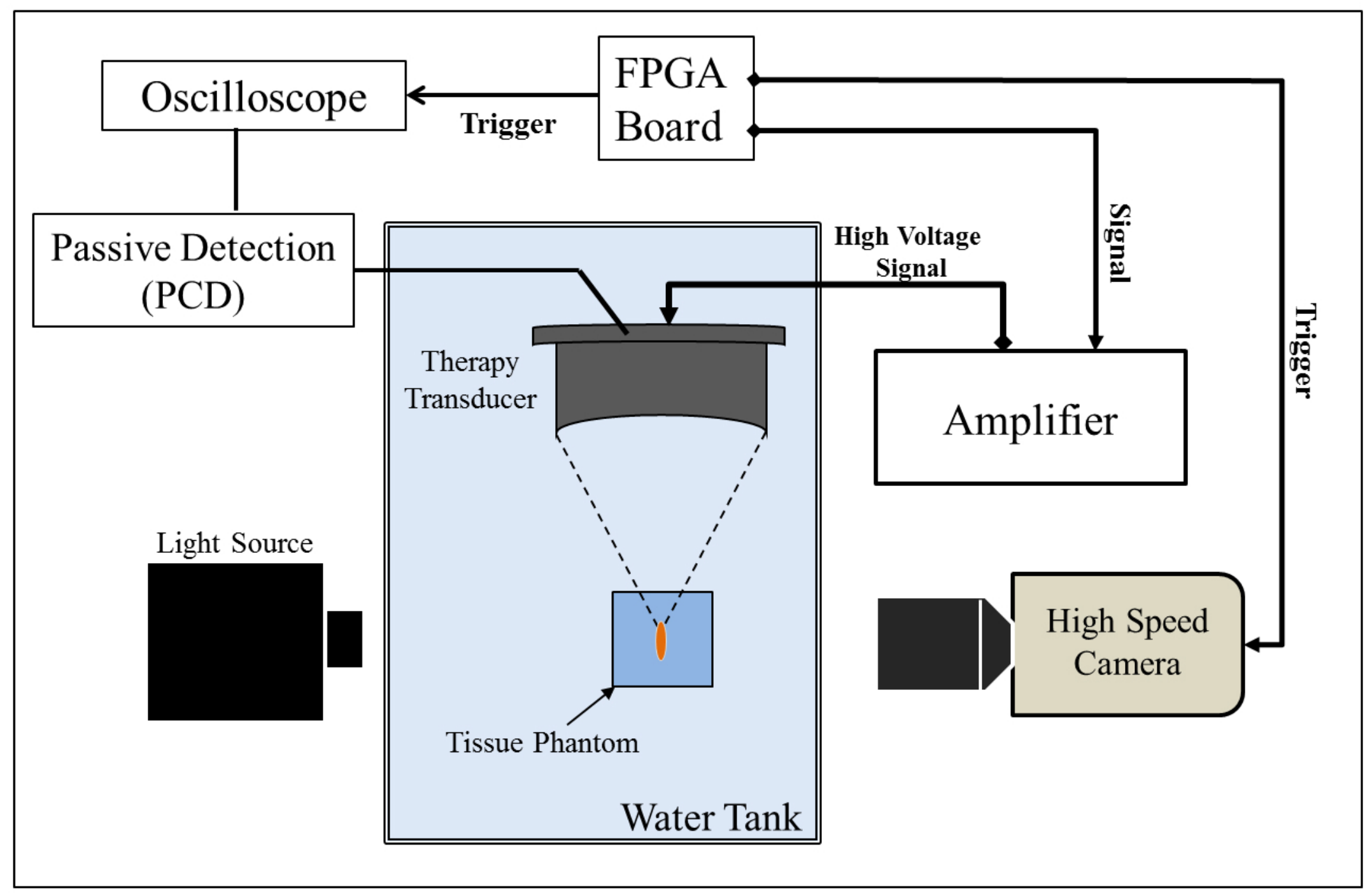


Figure 4
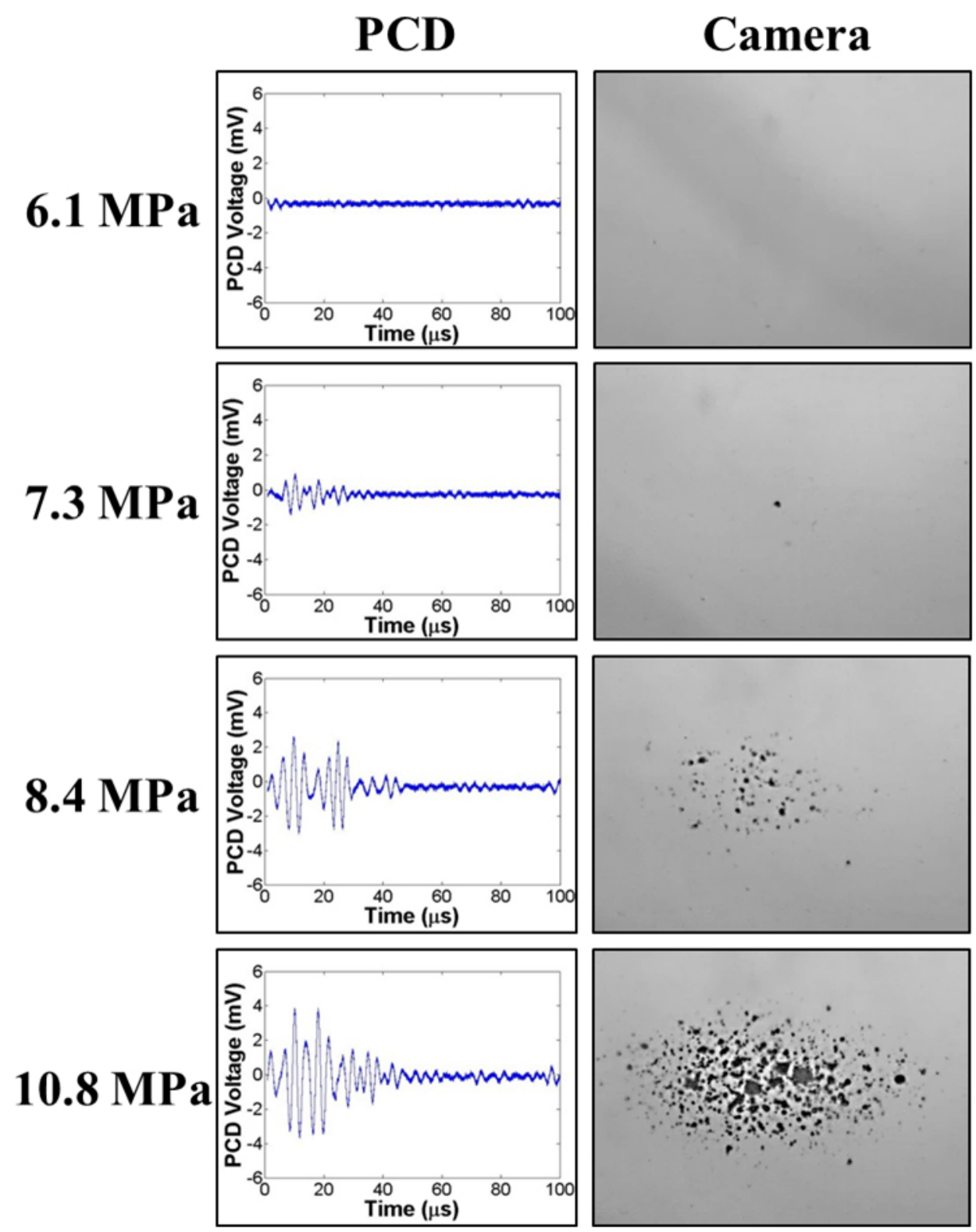
Figure 5

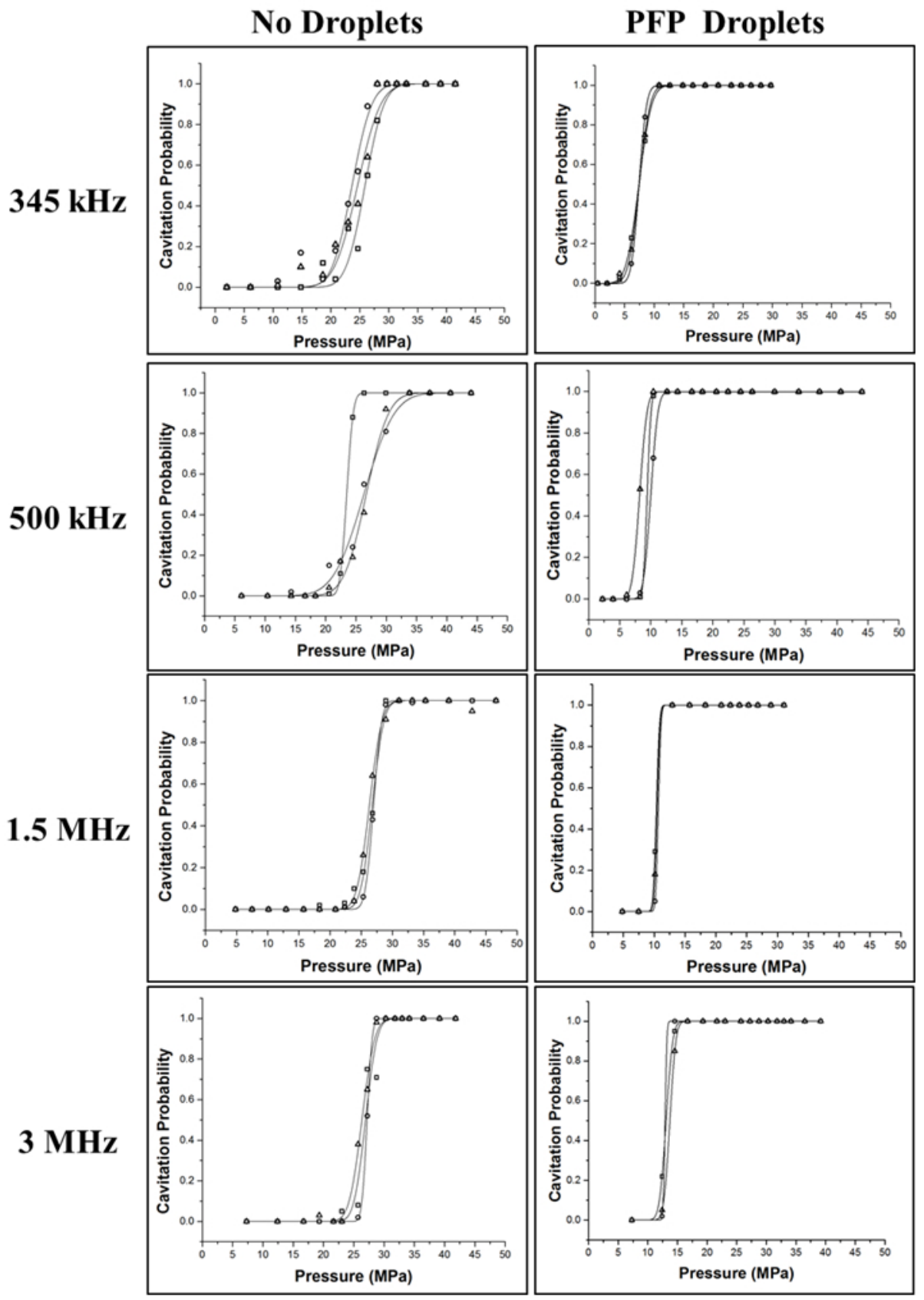




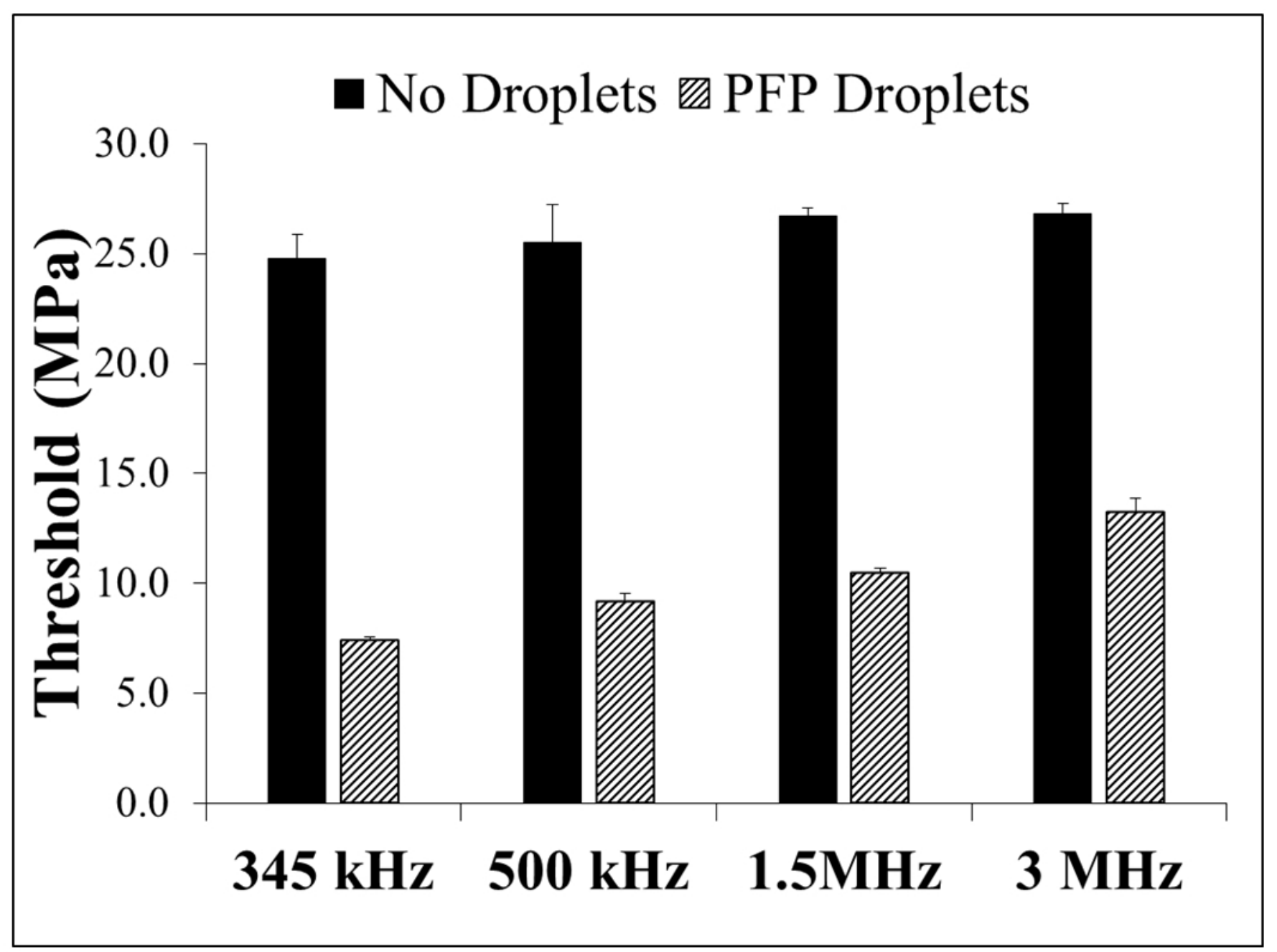




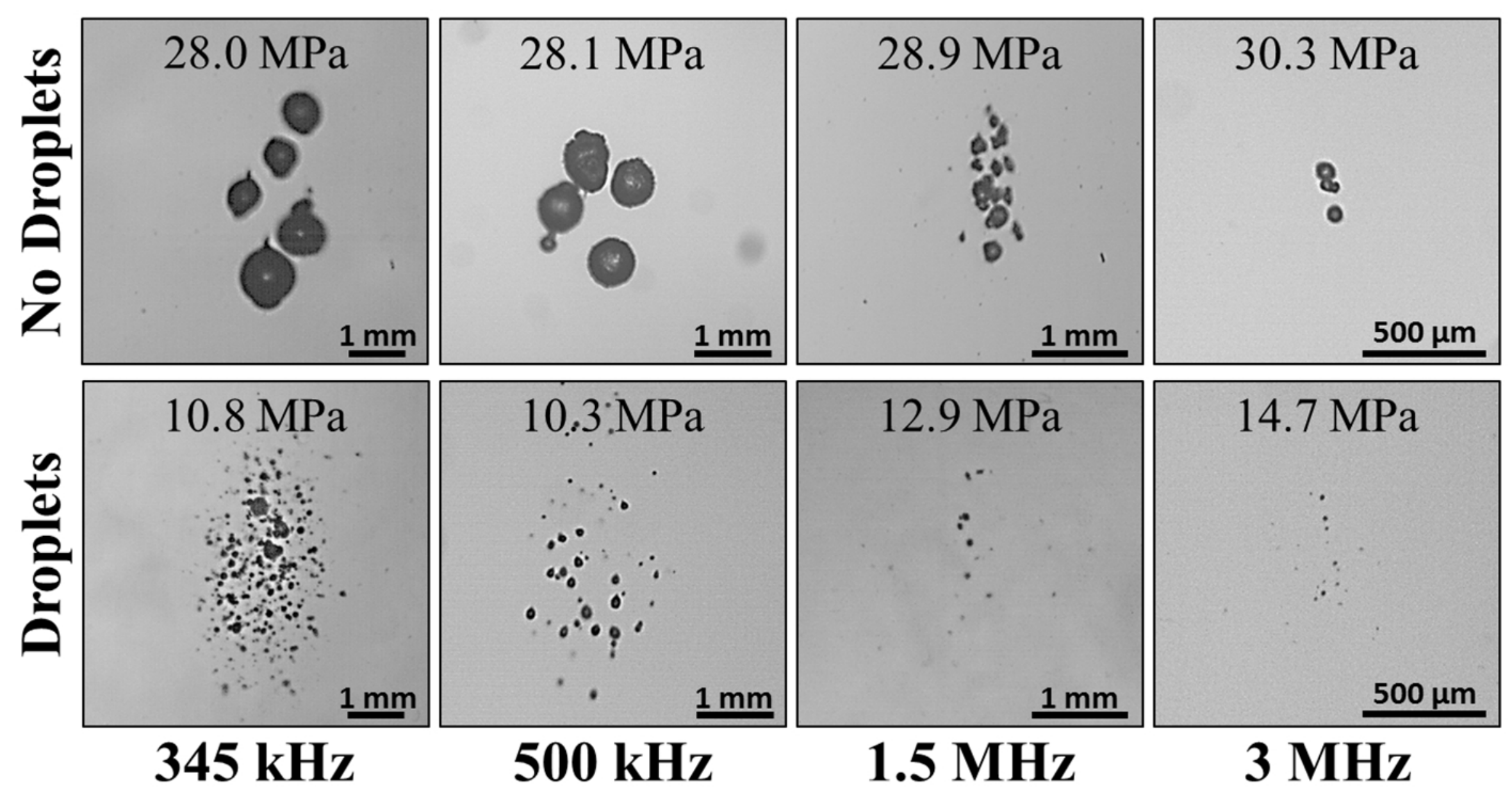




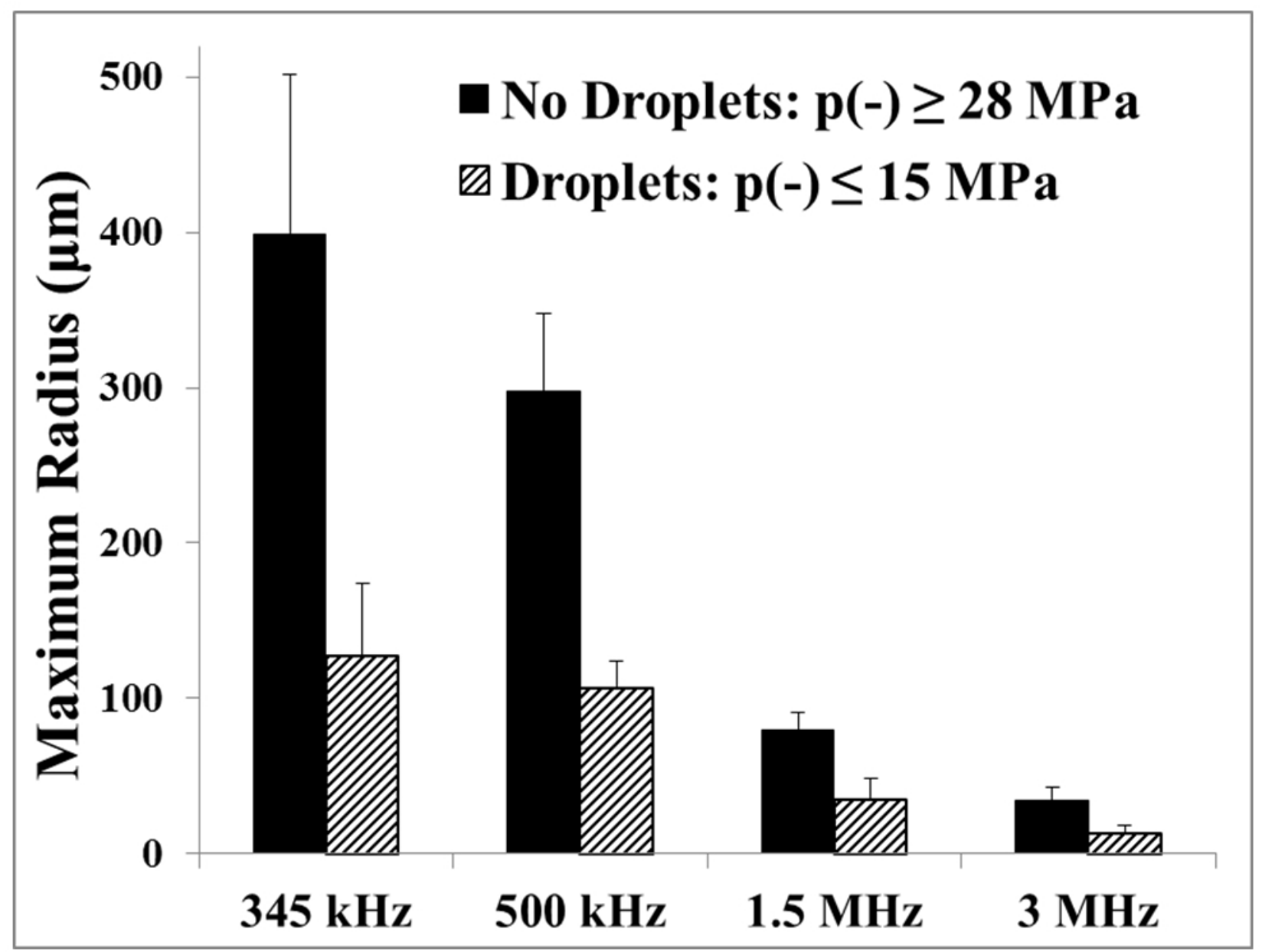




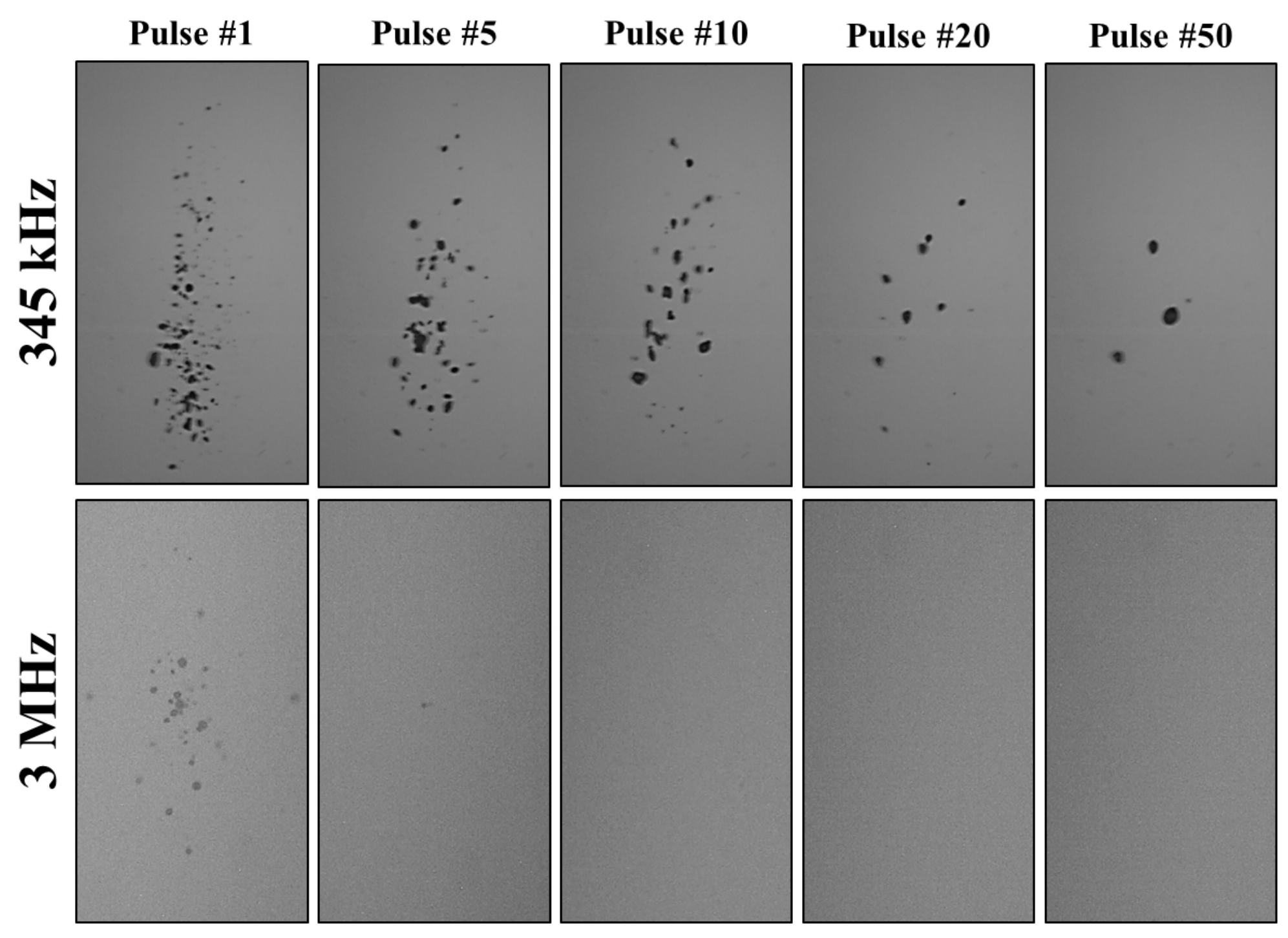


Figure 10

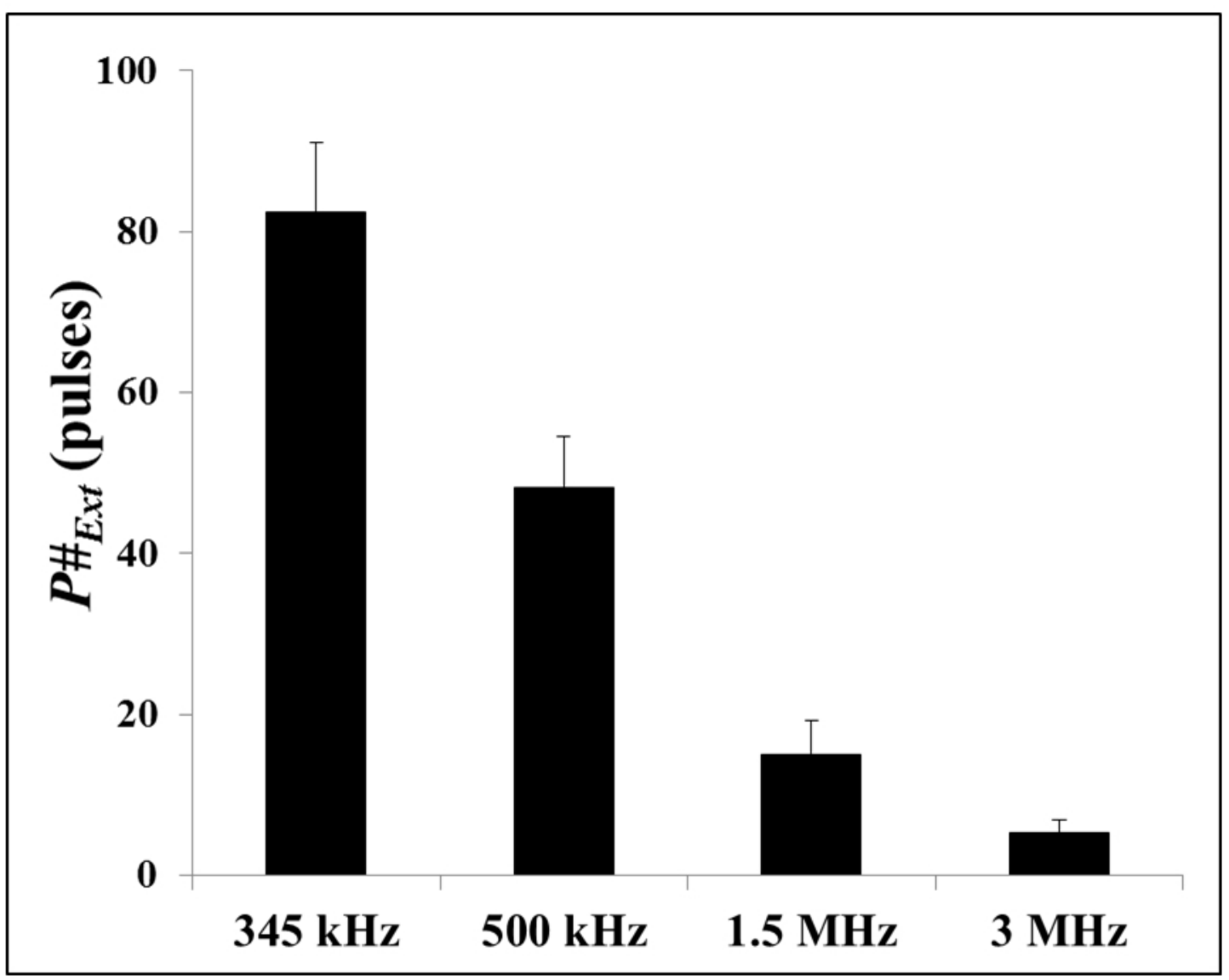

